\title{
Experimental and computational dynamic analysis of the foam concrete as a sub-base layer of the pavement structure
}

\author{
Veronika Valaskova ${ }^{1}$, Jozef Vlcek $^{2, *}$, and Marian Drusa ${ }^{2}$ \\ ${ }^{1}$ University of Zilina, Faculty of Civil Engineering, Department of Structural Mechanics and Applied \\ Mathematics, Univerzitna 8215/1, 01026 Zilina, Slovak Republic \\ ${ }^{2}$ University of Zilina, Faculty of Civil Engineering, Department of Geotechnics, Univerzitna 8215/1, \\ 01026 Zilina, Slovak Republic
}

\begin{abstract}
Dynamic properties of the new materials represent the actual problem, which is solved at many departments in the world at this time. Foam concrete (FC) is a material that has wide application in the civil engineering structures. Currently, it's mainly used in the floor structures of the buildings but its usability can be much wider thanks to its specific properties. The foam concrete contains closed air pores, what achieves its low volume weight (density) and saving of material inputs. It's a building material with good mechanical properties and low thermal conductivity and at the same time with high-tech processing.

Nowadays, a research aimed at the application of the foam concrete in the pavement structures takes place at the University of Zilina. Foam concrete could be utilized as a sub-base layer at the road reconstructions, excavations or as a regular layer of the new constructed pavements.

The submitted paper is dedicated to the numerical simulation based on the Finite element method (FEM) in pursuance of the outputs of the experimental investigation of the dynamic effect on the pavement structure which contains the foam concrete layer. Obtained dynamic parameters will be a background for the pavement design using the foam concrete.
\end{abstract}

\section{Introduction}

Foam concrete (FC), as a mixture of cement, water, additives and technical foam, is in principle well known for more than 30 years. It is building material with good mechanical properties, low thermal conductivity, simple and even high technological treatment. Foam concrete contains closed void pores what allows to achieve low bulk density and spare of input materials. Thanks to its properties, it is usable as a replacement of conventional subbase layers of the industrial floors, the transport areas or as a part of the foundation structures of the buildings. Mixture for the foam concrete can be prepared for various bulk densities. Today, we deal with bulk densities from 300 to $900 \mathrm{~kg} / \mathrm{m}^{3}$.

Nowadays, a research aimed at the application of the foam concrete in the pavement structures takes place at the University of Zilina. Foam concrete could be utilized as a subbase layer at the road reconstructions, excavations or as a regular layer of new constructed

\footnotetext{
${ }^{*}$ Corresponding author: j.vlcek@fstav.uniza.sk
} 
pavements $[1,2,3]$. Following the preparation of the large scale physical tests, preliminary numerical simulations will be carried out to identify the boundary condition of the future model. Very close distance to the source of the loading leads to consider the dynamic effects of the traffic load on the sub-base layer made of foam concrete. Relevant dynamic parameters such as dynamic modulus of elasticity or damping should be determined. This paper is dedicated to the Rayleigh damping characteristics of the foam concrete.

\section{Material damping}

The damping in engineering practice is defined as a reduction of the movement caused by the passive resistance of the material. It's the ability of the system to ensure the energy dissipation when structure is moving. During the dissipation, the kinetic energy changes to other types of energy that dispersed into the structure. This phenomenon causes the attenuation of the vibrations and impacts what provides a certain safety level of the structure except the specific situations such as the resonance.

The actual level of damping can be established through empirical approaches or calculations which require inputs determined by the experimental way. The introduction of the damping into the motion equations is always a compromise between the physical formulation and the requirements of the mathematical simplicity, [4].

Rayleigh damping is a convenient way to take into account the damping of particular materials of the model in the numerical simulations but determining the relevant parameters with sufficient reliability requires experimental measurements, [5].

Due to complexity of the large scale physical models, separated foam concrete specimens were tested in the laboratory to obtain foam concrete parameters at known boundary conditions.

\subsection{Rayleigh damping}

Mass and stiffness proportional damping, normally referred to as Rayleigh damping, is commonly used for linear and nonlinear dynamic analysis. It's suitable for an incremental approach of a numerical solution. In discrete systems, especially with regard to FEM application, Rayleigh's damping is calculated. Rayleigh damping is expressed using a Rayleigh damping matrix which is given by:

$$
[\mathbf{b}]=\alpha \cdot[\mathbf{m}]+\beta \cdot[\mathbf{k}],
$$

where $[\mathbf{m}]$ is a mass matrix and $[\mathbf{k}]$ is a stiffness matrix. The first member on the right side of the equation expresses attenuation proportional to the displacement velocity. The second member expresses attenuation of the velocity of the deformation, [6].

By multiplying the equation (1) to the left by the $\left[\mathbf{R}^{\mathbf{T}}\right]$ and the right $[\mathbf{R}]$, where $[\mathbf{R}]$ is the orthonormal modal matrix, we obtain:

$$
\begin{gathered}
{\left[\mathbf{R}^{\mathrm{T}}\right] \cdot[\mathbf{b}] \cdot[\mathbf{R}]=\alpha \cdot\left[\mathbf{R}^{\mathrm{T}}\right] \cdot[\mathbf{m}] \cdot[\mathbf{R}]+\beta \cdot\left[\mathbf{R}^{\mathrm{T}}\right] \cdot[\mathbf{k}] \cdot[\mathbf{R}],} \\
2\left[\omega_{\mathbf{b}}\right]_{\mathbf{D}}=\alpha \cdot[\mathbf{E}]_{\mathbf{D}}+\beta \cdot\left[\boldsymbol{\omega}_{\mathbf{j}}{ }^{2}\right]_{\mathbf{D}} .
\end{gathered}
$$

Because the i-th equation in (3) has a shape:

$$
2 \zeta_{i} \cdot \omega_{i}=\alpha+\beta \cdot \omega_{i}^{2}
$$

the damping ratio $\zeta_{i}$ for the i-th mode is:

$$
\zeta_{i}=1 / 2 \cdot\left[\left(\alpha / \omega_{i}\right)+\beta \cdot \omega_{i}\right]
$$


If the damping ratios for the $\mathrm{i}$-th and $\mathrm{j}$-th modes are $\zeta_{i}$ and $\zeta_{j}$, then the Rayleigh coefficients $\alpha$ and $\beta$ are calculated from the solution of the two algebraic equations:

$$
\frac{1}{2}\left[\begin{array}{ll}
1 / \omega_{i} & \omega_{i} \\
1 / \omega_{j} & \omega_{j}
\end{array}\right]\left\{\frac{\alpha}{\beta}\right\}=\left\{\frac{\zeta_{i}}{\zeta_{j}}\right\}
$$

The value of material damping is often not readily available. It requires some tests to properly calculate damping ratio. The tests should be properly executed because damping property depends on many parameters such as material property, geometry, temperature, etc.

\subsection{Experimental determination of coefficients $\alpha$ and $\beta$}

To calculate both the Rayleigh coefficients, two damping ratios with corresponding frequencies need to be known. If we assume that these frequencies are the natural frequencies of the dynamic system, we must excite the system with specific way to obtain the peaks for the first significant vibration modes. This could be sometimes difficult to achieve so another approach was adopted.

There are two methods to measure material damping ratio: logarithmic decrement method and half-power bandwidth method. In this case a half-power bandwidth method was selected because only one successful peak is required and this peak can be linked to the dominant and not necessarily natural frequency. Frequency response function (FRF) of the system must be obtained to use this method. Hammer or shaker tests can be performed to obtain FRF. Peaks in FRF show natural or dominant frequencies of the system $\omega_{n}$. We can calculate two points that are corresponding to half power bandwidth, $3 \mathrm{~dB}$ down from the top of the peak, as shown in the figure below.

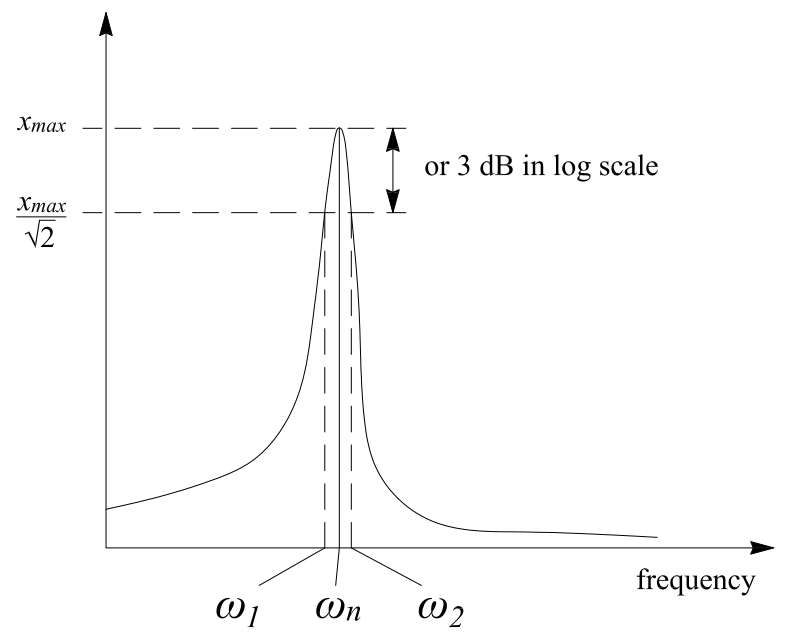

Fig. 1. Half-power bandwidth method.

The damping ratio $\zeta$ for the corresponding natural or dominant frequency $\omega_{n}\left(\omega_{i}\right.$ or $\left.\omega_{j}\right)$ can be calculated once the corresponding frequencies $\omega_{1}$ and $\omega_{2}$ are known. Material has more damping if frequency range between these two points is wider, [7].

The damping ratio can be expressed as: 


$$
\zeta=\frac{\omega_{2}-\omega_{1}}{2 \omega_{n}}
$$

The Rayleigh coefficients are given by:

$$
\begin{gathered}
2 \zeta_{i} \omega_{i}=\alpha+\beta \omega_{i}^{2}, 2 \zeta_{j} \omega_{j}=\alpha+\beta \omega_{j}^{2} \\
\alpha=\frac{2 \omega_{i} \omega_{j}\left(\zeta_{i} \omega_{j}-\zeta_{j} \omega_{i}\right)}{\omega_{j}^{2}-\omega_{i}^{2}}, \beta=\frac{2\left(\zeta_{j} \omega_{j}-\zeta_{i} \omega_{i}\right)}{\omega_{j}^{2}-\omega_{i}^{2}}
\end{gathered}
$$

\section{Results and discussion}

Required inputs for above mentioned calculations were obtained from experimental measurements in laboratory. A series of foam concrete specimens with age of 28 days were prepared, four of each nominal bulk density. The nominal dimensions of the specimens were $100 \times 105 \times 400 \mathrm{~mm}$. To obtain a frequency response function, each specimen was one-side bonded so cantilever with the free length of $300 \mathrm{~mm}$ was created and vertical hammer test was then performed.

\subsection{Experimental measurements}

The first three dominant frequencies were determined following the response of the foam concrete with the nominal bulk densities $300,500,700$ and $900 \mathrm{~kg} / \mathrm{m}^{3}$, fig. 2 .

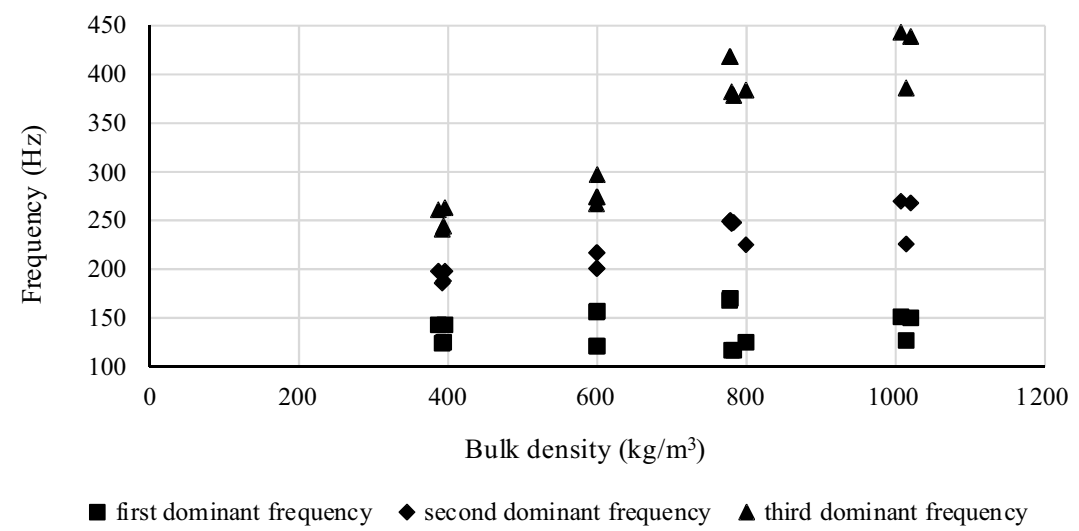

Fig. 2. The first three dominant frequencies.

Results indicate that the dominant frequencies reach higher values and larger gap between values from adjacent peaks with increasing bulk density. On the other hand, lower bulk densities show lower dispersion of the values for particular specimens.

Damping ratios were determined for the first two dominant frequencies and varied from 1.4 to $3.8 \%$ without significant dependency on the bulk density and with smaller dispersion of the values for the second dominant frequency.

Calculated values of the Rayleigh coefficients related to the measured bulk densities are plotted in the figures 3 and 4 . 


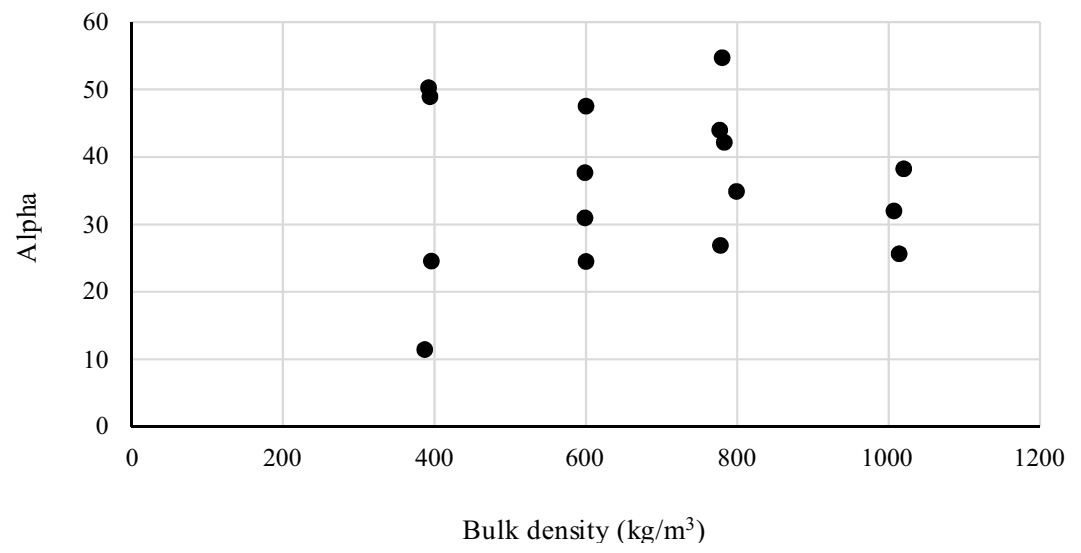

Fig. 3. Relation curves of alpha with measured bulk density.

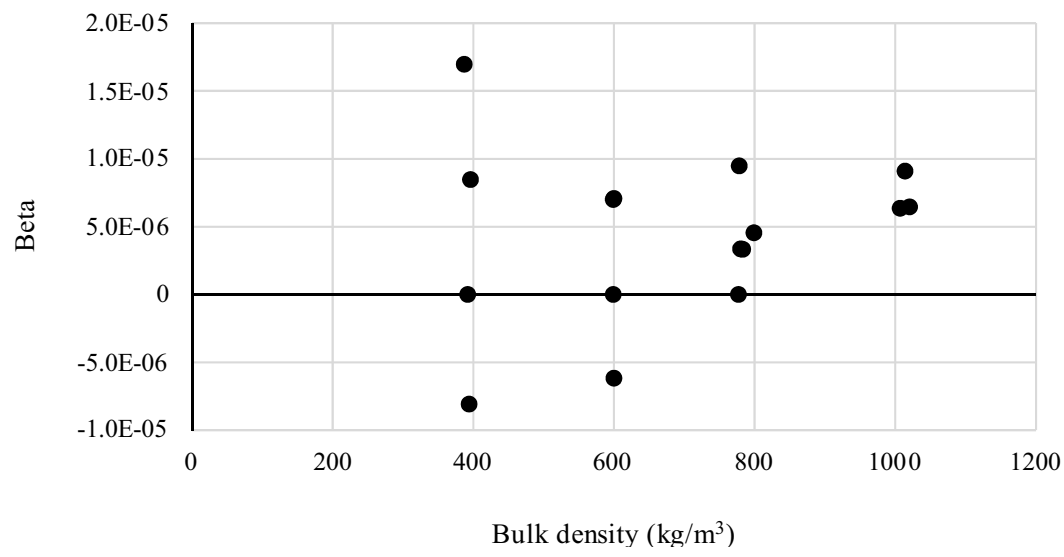

Fig. 4. Relation curves of beta with measured bulk density.

Results show large dispersion of the Rayleigh coefficients, especially of alpha at the lower bulk densities, but overall trend leads to smaller dispersion at higher densities. Generally, relatively high values of alpha and very small values for beta were reached. Alpha is damping proportional to the material mass. We assume that porous structure of the foam concrete allows to achieve higher damping what results in higher values of alpha. Beta is damping proportional to the material stiffness which is relatively low in case of the foam concrete. Obtained values of beta are very close to the zero value.

The results seem to be reliable in terms of overall trend but further measurements will be carried out to prove the accuracy of the reached values.

\subsection{Numerical simulation}

To verify the measured dominant frequencies of the specimen, numerical simulation in the software ADINA using the Finite Element Method (FEM) was carried out, [8]. At given Poisson's ratio of 0.25 , the dynamic modulus of elasticity was iterated via model calibration when the aim was to match the first dominant frequency obtained from the experiment. Linear elastic material model was adopted. The first three modes are plotted in the figure 5. 

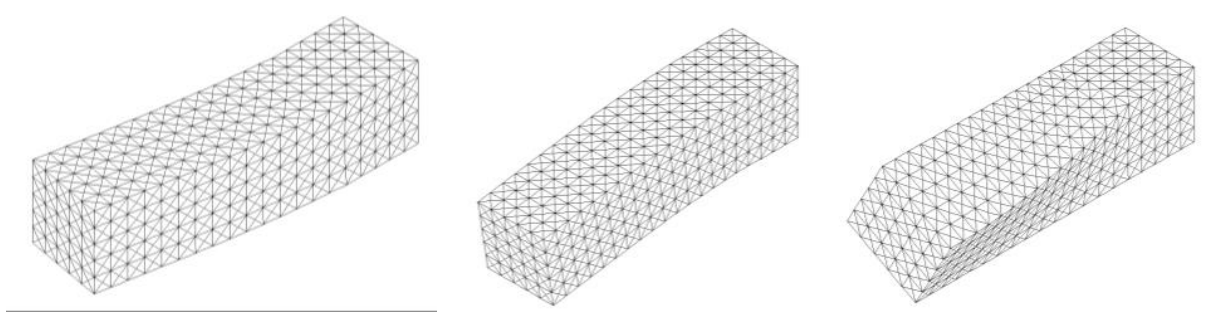

Fig. 5. First three modes of the specimen.

Model shows that the natural frequencies for the first two modes are very close to each other what is caused by the similar geometry of the specimen cross-section in both axes. The small gap between the first two natural frequencies is determined by the mass action in particular directions of vibration, fig. 5. Despite the matching of the peak related to the first two natural frequencies, others don't fit the frequency spectrum from the test. This phenomenon occurred because probably only second mode of the specimen was achieved during vertical load of the hammer test when mostly vertical vibrations took place. Frequencies from the test should be then considered as dominant frequencies rather than natural, fig. 2 .

\section{Conclusions}

The aim of the testing and simulation was to determine dominant frequencies for selected bulk densities of the foam concrete and calculate the Rayleigh coefficients that can be used for further analyses of the complex structures containing foam concrete.

Measured values of the Rayleigh coefficients indicate good damping potential of the foam concrete at lower frequencies what can be a benefit at the pavements in built-up areas.

Achieve results will be a background for further observations of the real scale physical model of the sub-base structure of the pavement.

This work was supported by the Scientific Grant Agency of the Ministry of Education of the Slovak Republic, Grant VEGA No. 1/0926/16.

\section{References}

[1] L. Izvolt et al., Mathematical modeling of the various factors impact on the frost depth of subgrade construction, XXIV R-S-P Seminar (2015)

[2] L. Izvolt et al., Calibration of TDR test probes for measuring moisture changes in the construction layers of the railway line, Proceedia Engineering 161 (2016)

[3] M. Kadela et al., Application of foamed concrete in road pavement - weak soil system, Procedia Engineering 193 (2017)

[4] J. Melcer et al, Dynamics of transport structures (Edis, Zilina, 2016)

[5] A. Herbut et al., Numerical modelling of Rayleigh wave propagation in course of rapid impulse compaction, IOP Conference Series 245 (2017)

[6] E. L. Wilson, Static and Dynamic Analysis of Structures (CaS, Berkeley, 2004)

[7] B. Olmos and J. Roesset, Evaluation of the half-power bandwidth method to estimate damping in systems without real modes, Earthquake Engineering \& Structural Dynamics, (2010).

[8] ADINA R\&D, Theory and Modeling Guide. Volume I: ADINA, ADINA R\&D (2012) 